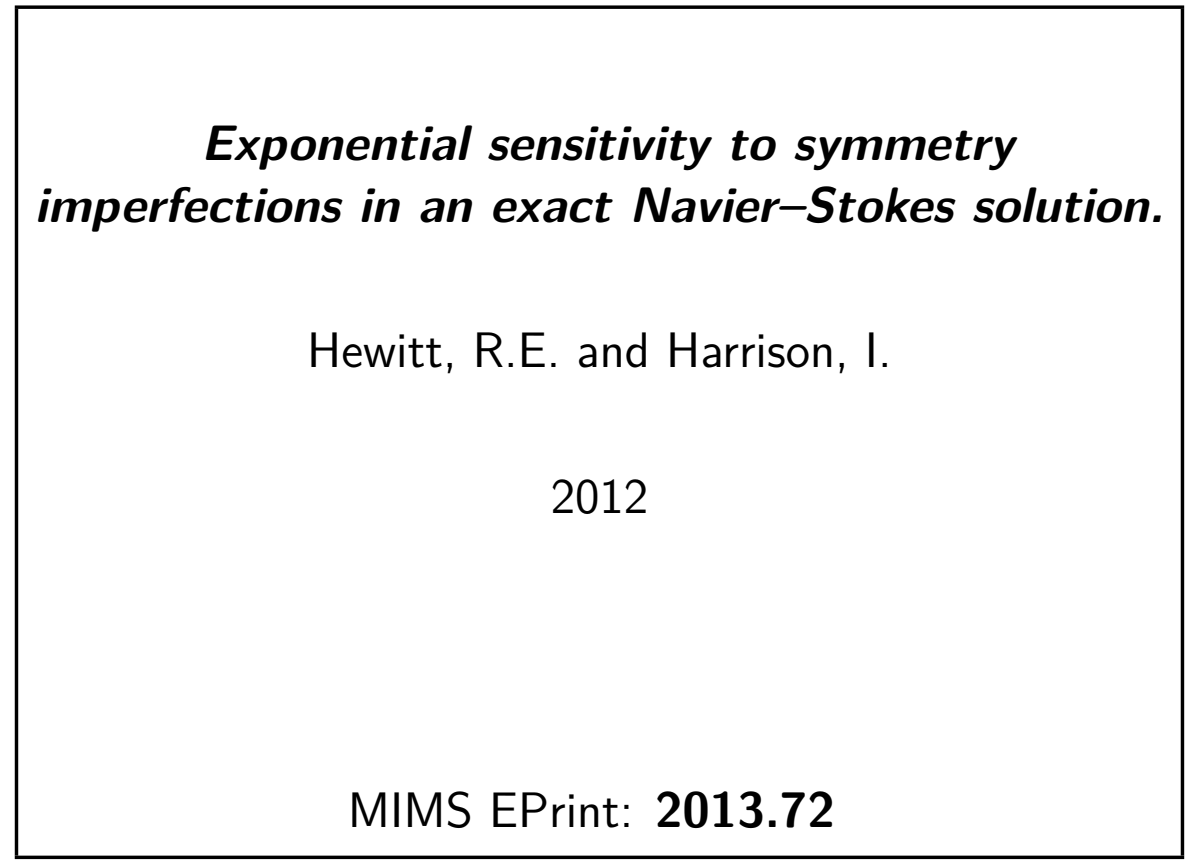

Manchester Institute for Mathematical Sciences

School of Mathematics

The University of Manchester

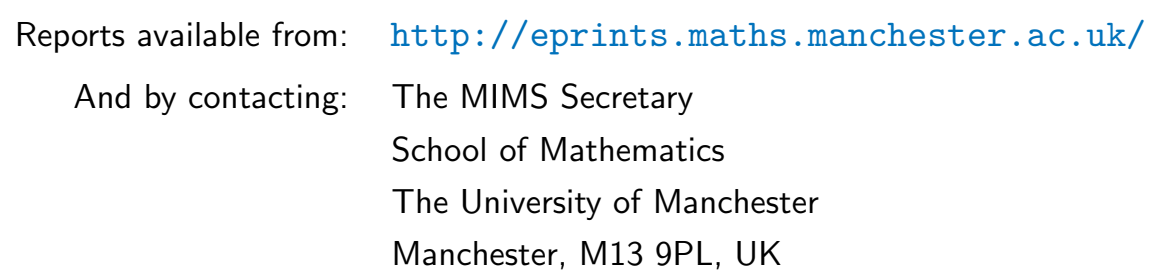

ISSN 1749-9097 


\title{
Exponential sensitivity to symmetry imperfections in an exact Navier-Stokes solution
}

\author{
Richard E. Hewitt • Iain Harrison
}

Received: 13 January 2011 / Accepted: 26 October 2011 / Published online: 18 November 2011

(C) Springer Science+Business Media B.V. 2011

\begin{abstract}
We consider the (radial) stretching flow of an incompressible viscous fluid between two parallel plates. For infinite plates, a well-known self-similar solution reduces the Navier-Stokes equations to a simple nonlinear boundary-value problem. We demonstrate that, for large Reynolds numbers, a naïve matched asymptotic description of the self-similar flow yields a continuum of solutions. To describe which of the continuum of states is realised requires the inclusion of terms that are beyond all orders in the asymptotic description. Sensitivity to exponentially small terms in the asymptotic description has practical significance in that (i) exponentially small symmetry imperfections in the boundary conditions have a leading-order effect, and (ii) linearised perturbations are seen to decay only on exponentially long space/time scales owing to the presence of eigenmodes that are exponentially near neutral. The results of axisymmetric Navier-Stokes computations are presented to show that the asymptotic description of the self-similar states (and their stability) is of practical relevance to finite-domain solutions.
\end{abstract}

Keywords Exact solutions $\cdot$ Exponential asymptotics $\cdot$ Stability $\cdot$ Stagnation-point flows

\section{Introduction and formulation}

Exact solutions to the incompressible Navier-Stokes equations have always been of great interest. Solutions of stagnation-point type, in which the velocity components vary at most linearly with distance from some line/axis of symmetry have been discussed in great detail. In these cases, the assumed self-similar stagnation form reduces the steady Navier-Stokes equations to a nonlinear boundary-value problem for a system of ordinary-differential equations. This class of solutions contains some of the most well-known and well-studied states, including the flows described by Berman [1] and Terril [2] (driven by suction/injection through porous walls), Karman [3] (driven by boundary rotation) and Brady and Acrivos [4] (driven by a stretching wall motion). The full history of these solutions is too extensive to describe here, and the interested reader is directed to the detailed discussion presented in the recent book by Riley and Drazin [5].

Exact stagnation-type solutions are of inherent interest in that, whilst simple and generic in form, they can also be extended to unsteady flows without breaking the self-similar form. In many cases, the resulting unsteady nonlinear problem exhibits interesting features, including non-uniqueness of steady states, limit points, pitchfork

R. E. Hewitt $(\varangle) \cdot$ I. Harrison

School of Mathematics, University of Manchester, M13 9PL Manchester, UK

e-mail: richard.e.hewitt@manchester.ac.uk 
Fig. 1 A schematic view of the flow geometry. The fluid motion is driven by the radial stretching of the bounding walls. The motion of each wall is towards the axis of symmetry, with a magnitude that is proportional to the distance from the axis

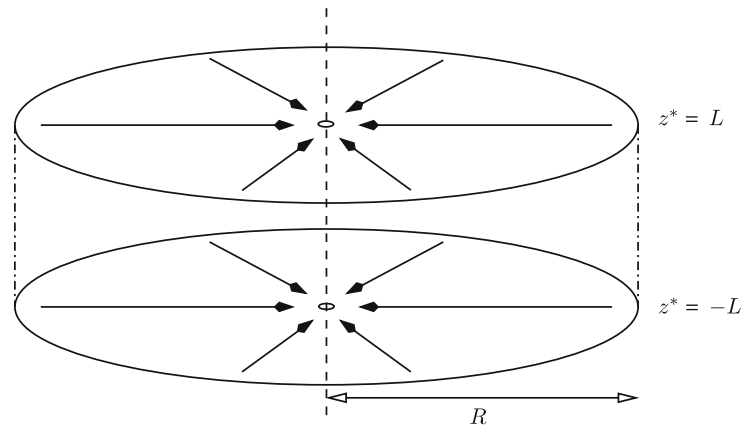

bifurcations, Hopf bifurcations, finite-time singularities and regions of parameter space in which no steady solution can be located.

The solutions of 'stretching type' are driven by a boundary motion that corresponds to the wall stretching in its own plane. In the current article, we consider the radial stretching problem, in which the flow is confined between two planes, each of which is in motion towards an axis of rotational symmetry as shown in Fig. 1. The speed of the wall motion is proportional to the distance from the axis of rotational symmetry. These flows may seem, at first sight, to be of less practical significance than the corresponding states driven by a simple rotation of, or transpiration through, a bounding wall, but they are of significance to steady-streaming problems. For example, these flows have application to problems in which bounding walls are in small-amplitude, high-frequency, periodic motion normal to their plane, which leads to a wall stretching rate that is related to the local streaming Reynolds number. An example of just such an application is the recent study of Hall and Papageorgiou [6], which considered a planar motion driven by periodic oscillation of two parallel walls. In their case, the sub-problem in the steady-streaming limit is an analogous planar stretching flow. The mid-plane symmetry breaking bifurcation of this planar stretching flow was shown to be crucial, since it is the subsequent Hopf bifurcation of the symmetry broken steady-streaming flow that is responsible for the quasi-periodic behaviour (and ultimately the subsequent chaotic motion). As we shall show in this study, the switch from a planar stretching flow to a radial stretching flow leads to a qualitative change in the solution structure, with the radial case being significantly more subtle.

The analysis we present herein is related to the well-known Berman-Terril-Robinson (BTR) problem for flow in a planar porous channel; see [7,8]. The BTR problem is one in which, in the inviscid limit, there are a number of discrete solution branches; however, two of the states only differ by terms that are exponentially small. Similar behaviour is also found for porous pipes, and the early article of Terrill [9] was an important step in recognising the influence of exponentially small terms in the asymptotic description. The BTR class of problems are not isolated cases and a thorough review of other situations (across a broad range of disciplines) that present comparable features was presented in [10].

For the flow under consideration here we show that the system behaves differently from the BTR problem. Rather than the exponentially small terms distinguishing between a pair of discrete solutions to the boundary value problem, we demonstrate that, to algebraic order, a continuum of solutions exists at large Reynolds numbers. For the high Reynolds number limit to result in a (locally) unique solution (selecting one solution from the 'algebraic continuum'), we show that terms beyond all orders must be included in the asymptotic description. This provides a mechanism for perturbations that are beyond all orders in the asymptotic series to determine the leading order response of the flow; a behaviour that, one may argue, is potentially more significant than that found in the BTR problem.

\subsection{Formulation}

We consider the axisymmetric flow between two parallel planes of infinite extent, separated by a distance $2 L$. Between the two boundaries is an incompressible, viscous fluid of density $\rho$ and kinematic viscosity $v$. A dimensional 
cylindrical polar coordinate system $\left(r^{*}, \vartheta, z^{*}\right)$ with velocity field $\left(u^{*}, 0, v^{*}\right)$ is centred midway between the planes on the axis of symmetry, such that the boundaries are at $z^{*}= \pm L$, and the system is (notionally) of radial extent $R$. The boundaries undergo a stretching motion in such a way that the radial, dimensional velocity $u^{*}$ on $z^{*}= \pm L$ is $u^{*}\left(r^{*}, \pm L\right)=-C^{ \pm} L^{-1} r^{*}$, for constants $C^{ \pm}$. As noted above, whilst such a boundary condition might not appear useful at first sight, it is applicable to a subset of broader practical problems. One important case arises in steady-streaming flows, for which the wall speed constants $C^{ \pm}$will be related to the local streaming Reynolds number.

Following the introduction of the dimensionless coordinates $r^{*}=R r, z^{*}=L z$, velocities $u^{*}=A C^{-} u(r, z, t)$, $v^{*}=C^{-} v(r, z, t)$ and pressure $p^{*}=\rho C^{-2} A^{2} p(r, z, t)$, the governing equations become

$u_{t}+u u_{r}+v u_{z}=-p_{r}+\frac{1}{R e}\left\{u_{z z}+\frac{1}{A^{2}}\left(u_{r r}+\frac{1}{r} u_{r}-\frac{u}{r^{2}}\right)\right\}$,

$v_{t}+u v_{r}+v v_{z}=-A^{2} p_{z}+\frac{1}{R e}\left\{v_{z z}+\frac{v_{r r}}{A^{2}}\right\}$,

$u_{r}+\frac{u}{r}+v_{z}=0$,

where the Reynolds number is $R e=C^{-} L / v$ and $A$ is an aspect ratio of the domain defined to be $A=R / L$. The boundary conditions are $u(r, z=-1)=-r, u(r, z=1)=-C^{+} r / C^{-}$and $v(r, z= \pm 1)=0$.

There is a well-known self-similar solution for the flow in the stagnation-point form:

$(u(r, z, t), v(r, z, t))^{T}=(r U(z, t), V(z, t))^{T}$,

$p(r, z, t)=r^{2} \frac{P(t)}{2}+\frac{1}{A^{2}} Q(z, t)$.

This solution is exact in the framework of the Navier-Stokes, that is, the terms proportional to $A^{-2}$ in (1) are identically zero, and no approximations are required. However, we may also view this solution as a leading-order (boundary-layer type) solution for domains of large aspect ratio $A \gg 1$. To apply (2) to 'large' domains requires us to neglect any far-field conditions at $r^{*}=R$ under the common assumption that they are 'sufficiently far away' to not impact the solution elsewhere. We will address this assumption later in this article through a combination of a spatial stability analysis and computation of solutions to (1) at finite values of $A$.

If we restrict attention for the moment to steady self-similar solutions, then we are required to solve a deceptively simple fourth-order boundary-value problem for the velocity components $U(z), V(z)$ and pressure constant $P$ :

$$
\begin{aligned}
& \frac{U^{\prime \prime}}{R e}=P+U^{2}+V U^{\prime}, \\
& 2 U+V^{\prime}=0,
\end{aligned}
$$

where

$U(-1)=-1, \quad U(1)=C^{+} / C^{-} \equiv-(1+\Delta), \quad$ and $\quad V( \pm 1)=0$.

Here the prime notation indicates differentiation with respect to the dimensionless axial coordinate $z$ and $\Delta$ is a measure of asymmetry in the wall boundary conditions. Throughout this study, we concentrate on the case where any asymmetry in the boundary conditions is small.

\section{Numerical solutions for steady self-similar states}

We begin by presenting some brief (self-similar) numerical results, which we shall use to motivate the subsequent asymptotic descriptions. Numerical solutions to (3) have been obtained by several methods, which were all in agreement. For the figures presented herein, we used a finite-difference representation of the governing equations, writing the system as four first-order equations and evaluating the equations at mid-nodal points with a second-order 


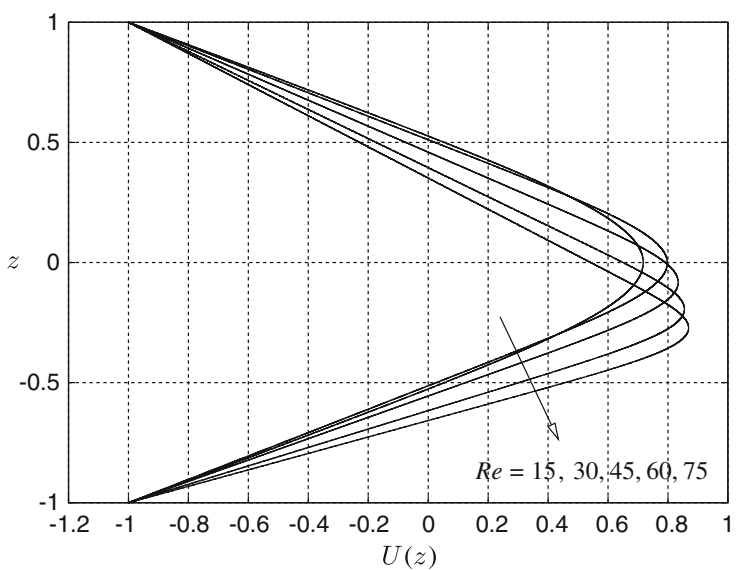

Fig. 2 Profiles of the radial component $U(z)$ for increasing Reynolds number with a small imperfection of $\Delta=10^{-6}$. We note the dramatic loss of a mid-plane symmetric (about $z=0$ ) profile for $R e=45,60,75$

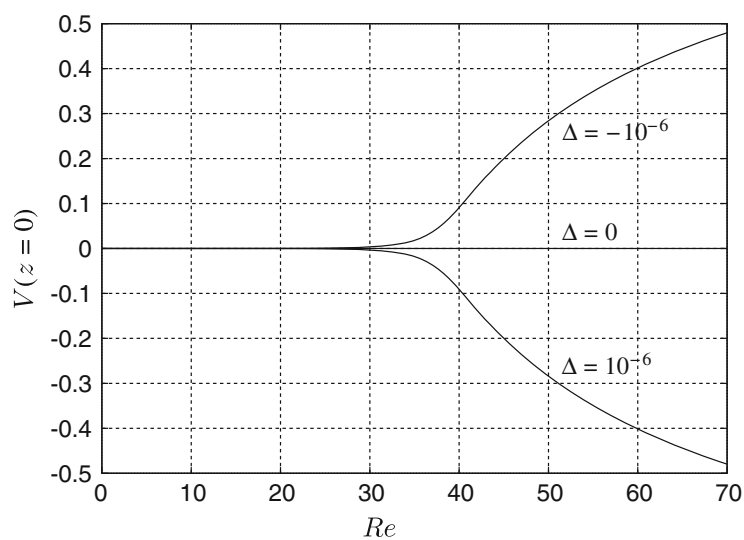

Fig. 3 Computations for increasing $R e$ with an imperfection in the symmetry of the wall boundary conditions of $\Delta= \pm 10^{-6}$. A symmetric profile is maintained for all $R e$ when $\Delta=0$, for which $V(z=0)=0$. Despite the qualitative appearance of a softened pitchfork bifurcation, we emphasise that this is not the case, and there is no bifurcation

accurate scheme. The nonlinearity is handled by Newton iteration and all tolerances were set to $10^{-10}$. Computational meshes ranged up to $8 \times 10^{4}$ points across the domain; the need for this very large number of points (for a simple boundary value problem) is associated with a subtle sensitivity of the system to asymmetry about $z=0$.

Numerical difficulties (convergence failure) can occur when attempting to solve the problem for $\Delta=0$ unless the symmetry is enforced by solving over the half-domain $z \in[0,1]$. As we shall explain herein, this difficulty is a feature of the solution set of (3), the source of which lies in the existence of a continuum of states that are parameterised by exponentially small differences in the symmetry of the boundary conditions. Similarly, if one monitors the determinant of the Jacobian matrix during the solution procedure to detect bifurcation points, then spurious bifurcations will typically be observed.

Solution of the imperfect problem $(\Delta \neq 0)$ is a more straightforward process, and results are shown in Figs. 2, 3, 4 and 5. It is immediately apparent, see Fig. 2, that the radial velocity profile becomes skewed at sufficiently large values of the Reynolds number, even for small symmetry imperfections. It is worth noting at this stage that the radial flow is bi-directional (owing to the obvious mass conservation constraint), and this makes the linear stability problem (as discussed later in this study) a little more challenging than would be the case for uni-directional flows.

No computational difficulties are encountered when beginning at, for example, $R e=1$ with $\Delta=10^{-6}$, and then using a continuation procedure to progress to larger values of $R e$. However, any subsequent attempt to continue these solutions back to $\Delta=0$ can lead to convergence difficulties at larger values of $R e$.

At first sight, from the results of Fig. 3, one might believe that there is a singular point on the symmetric solution branch (near $R e \approx 40$ ) that corresponds to a pitchfork bifurcation and a loss of mid-plane symmetry. However, although Fig. 3 has qualitative features in line with a disconnected (softened) pitchfork bifurcation, our later asymptotic description will demonstrate that no such bifurcation exists and the symmetric solution branch is locally unique. This uniqueness is demonstrated in the results of Figs. 4 and 5. Figure 4 demonstrates that mid-plane symmetric flow is indeed recovered (smoothly) at $R e=50$, but only for symmetry imperfections of $\Delta \lesssim 10^{-8}$. Similarly in Fig. 5, we present $V(z=0)$ as the obvious measure of asymmetry in the flow as a function of the imperfection $\Delta$ for a range of Reynolds numbers. As is clearly seen, for $R e=60$ we obtain what appears to be very close to a continuum of states near $\Delta=0$, with $-0.4<V(z=0)<0.4$. However, a view of Fig. 5 over a sufficiently small range of $\Delta$ shows that $V(z=0)$ remains single valued, but the slope of the data is exponentially increasing with $R e$. 


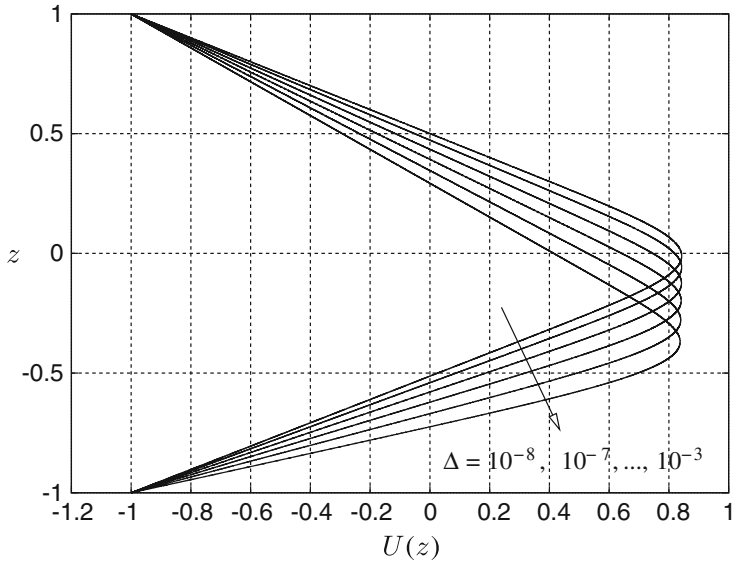

Fig. 4 Profiles of $U(z)$ for $\Delta=10^{-8}, 10^{-7}, \ldots, 10^{-3}$ for fixed $R e=50$. It is clear that imperfections in the symmetry of the boundary conditions have a dramatic influence on the $O(1)$ velocity components. Furthermore, we note the passive nature of the near wall regions

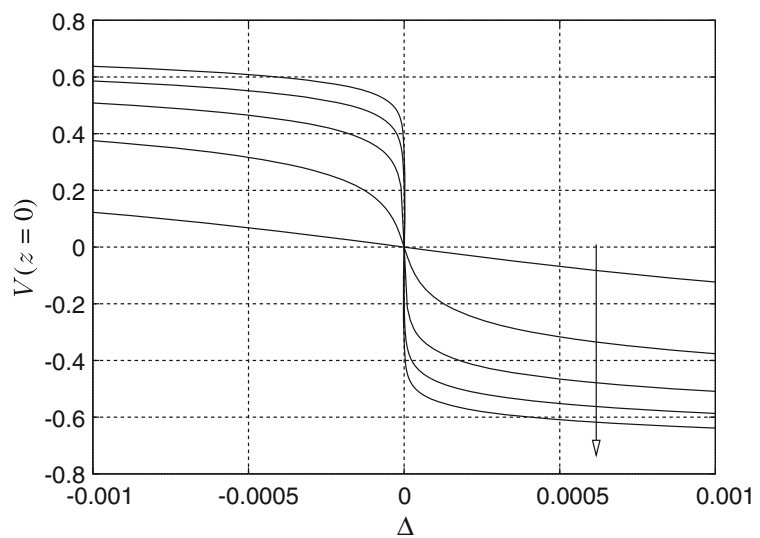

Fig. 5 A (mid-plane symmetry) measure, the normal velocity $V$ evaluated at the midplane $z=0$, as a function of the imperfection parameter $\Delta$ for a range of Reynolds number $R e=$ 20,30, 40, 50, and 60 (increasing in the direction of the arrow shown). For increasing $R e$, the system is clearly highly sensitive to $\Delta$; however, $V(z=0)$ remains single-valued as a function of $\Delta$

The origin of all the numerical features described above will be clarified below by an asymptotic investigation of the high-Reynolds number structure of the solutions. As we shall show, convergence difficulties are to be anticipated in that, as $R e \rightarrow \infty$, there exists an $O(1)$ sensitivity to exponentially small, $O\left(R e^{-\frac{1}{2}} \exp (-\mu R e)\right)$ perturbations to the symmetry of the boundary conditions; here $0<\mu<\frac{1}{3}$ is a parameter to be described later. It is worth recalling here that since this is an exact solution of the Navier-Stokes equations, these features must also be present in the broader context of the full governing equations for at least a subset of boundary conditions applied at the edge $\left(r^{*}=R\right)$ of the domain.

\section{A perturbation expansion for self-similar steady states as $\boldsymbol{R} \boldsymbol{e} \rightarrow \infty$}

On the basis of Fig. 4, we conjecture that the large Reynolds number structure consists of two inviscid regions $z \in[-1, c]$ and $z \in[c, 1]$ with a viscous shear layer at $z=c$. We note that there is little evidence of any structure near the walls at $z= \pm 1$, and we shall assume from the outset (as suggested by the results of Fig. 5) that the measure of the imperfection $\Delta \ll R e^{-k}$ for any positive value $k$. A schematic of the proposed structure is shown in Fig. 6.

\subsection{Inviscid regions: $z \in[-1, c]$ and $z \in[c, 1]$}

For large- $R e$, we seek an outer perturbation expansion in the form:

$$
\begin{aligned}
& U=U_{0}^{ \pm}+U_{1}^{ \pm} R e^{-1}+\cdots, \\
& V=V_{0}^{ \pm}+V_{1}^{ \pm} R e^{-1}+\cdots, \\
& P=P_{0}^{ \pm}+P_{1}^{ \pm} R e^{-1}+\cdots,
\end{aligned}
$$

where the terms $(\cdot)_{i}^{+}$terms are the expansion components in $z \in[c, 1]$ and the terms $(\cdot)_{i}^{-}$terms are the corresponding terms in the region $z \in[-1, c]$. 


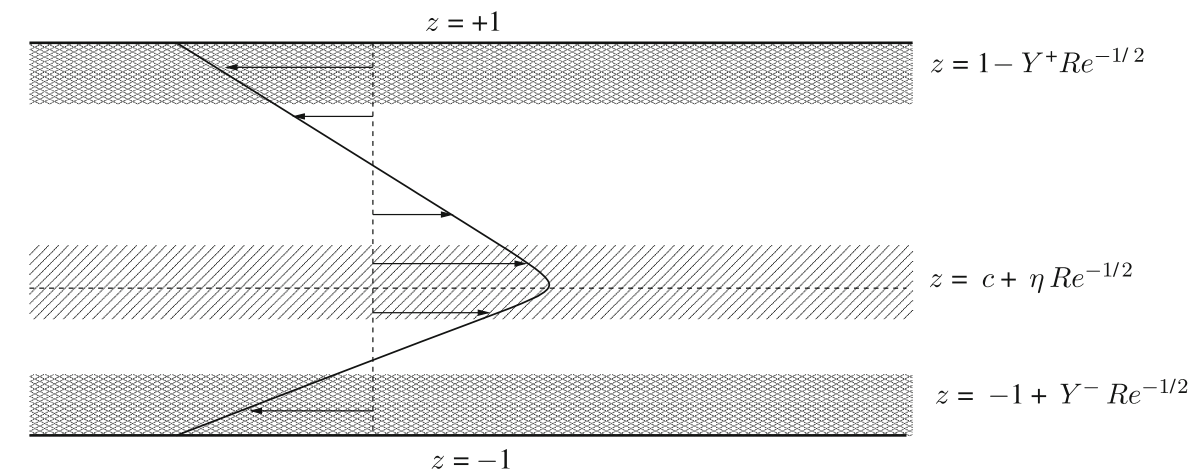

Fig. 6 A schematic view of the asymptotic regions as $R e \rightarrow \infty$. Here the viscous wall layers spanned by the rescaled coordinates $Y^{ \pm}$ only need to be considered for terms that become exponentially small for increasing Reynolds number

In the lower inviscid region, this leads to

$P_{0}^{-}+U_{0}^{-2}+V_{0}^{-} U_{0}^{-\prime}=0$

$2 U_{0}^{-}+V_{0}^{-\prime}=0$.

In the absence of any leading-order near-wall structure at $z=-1$, solving subject to $U_{0}^{-}(-1)=-1, V_{0}^{-}(-1)=0$ and $V_{0}^{-}(c)=0$ yields

$V_{0}^{-}(z)=\frac{2(c-z)(1+z)}{c+1}, \quad U_{0}^{-}(z)=\frac{2 z+1-c}{c+1}, \quad P_{0}^{-}=-1$.

The same process in the region $z \in[c, 1]$ provides

$V_{0}^{+}(z)=\frac{2(c-z)(z-1)}{c-1}, \quad U_{0}^{+}(z)=\frac{2 z-1-c}{c-1}, \quad P_{0}^{+}=-1$.

3.2 The viscous shear layer: $z=c+\eta R e^{-1 / 2}$

This viscous inner layer straddles the point $z=c$. In this region, given the nature of the outer solutions, we seek an expansion in the form:

$U=1+\tilde{U}_{s 1}(\eta) R e^{-1 / 2}+\cdots$,

$V=-2 \eta R e^{-1 / 2}+\tilde{V}_{s 1}(\eta) R e^{-1}+\cdots$.

At leading order, we obtain

$\tilde{U}_{s 1}^{\prime \prime}=2 \tilde{U}_{s 1}-2 \eta \tilde{U}_{s 1}^{\prime}$,

$2 \tilde{U}_{s 1}+\tilde{V}_{s 1}^{\prime}=0$,

subject to matching with the outer inviscid solutions above as $\eta \rightarrow \pm \infty$. The appropriate solution is

$$
\begin{aligned}
& \tilde{U}_{s 1}(\eta)=2\left(\eta[c+\operatorname{erf}(\eta)]+\frac{e^{-\eta^{2}}}{\sqrt{\pi}}\right) /\left(c^{2}-1\right), \\
& \tilde{V}_{s 1}(\eta)=-\eta \tilde{U}_{s 1}(\eta)-\operatorname{erf}(\eta) /\left(c^{2}-1\right) .
\end{aligned}
$$

The viscous shear layer's reduction in radial mass flow results in a correction to the inviscid region of $O\left(R e^{-1}\right)$. Continuing our expansion further reveals the additional corrections in the outer inviscid problems. These are straightforward to determine following matching with the above shear-layer solution: 
$V_{1}^{-}(z)=\frac{(1+z)^{2}}{(1+c)^{3}(c-1)}, \quad U_{1}^{-}(z)=-\frac{1+z}{(1+c)^{3}(c-1)}, \quad P_{1}^{-}=0$,

and

$V_{1}^{+}(z)=-\frac{(z-1)^{2}}{(c-1)^{3}(c+1)}, \quad U_{1}^{+}(z)=\frac{z-1}{(c-1)^{3}(c+1)}, \quad P_{1}^{+}=0$.

\subsection{The influence of the boundary layers}

Clearly in the expansion thus far, there is no role for the imperfection $\Delta$ since we have chosen it to be beyond all orders in magnitude. Furthermore, we may observe that there is no mechanism for determining the constant $c$ in the leading-order solution. In fact, to the order we have described here, we must conclude that there is a continuum of solutions parameterised by the value of $c$. We conjecture at this stage that continuing to higher order in the hierarchical expansion scheme outlined above does not, at any algebraic order, act to determine a unique value of $c$.

In this section, we will show that the value of $c$, which effectively determines the shape of the leading-order velocity profiles as shown in Figs. 2 and 4, is fixed by the beyond all orders imperfection $\Delta$. To demonstrate this, we must consider the influence of boundary layers at $z= \pm 1$, even though these boundary layers are only non-trivial for terms that are exponentially small. The concept of a boundary layer is rather less applicable to exponentially small terms, but this discussion is nonetheless useful in motivating a later WKB technique that is more global in nature.

\subsubsection{A wall layer at $z= \pm 1$}

The radial momentum equation, when evaluated at either wall, determines the pressure constant to be

$P=\left.\frac{1}{R e} U^{\prime \prime}\right|_{z= \pm 1}-\left.U^{2}\right|_{z= \pm 1}$.

Because the upper wall boundary condition is $U(1)=-(1+\Delta)$, the proposed form for the pressure is

$P=-1+\bar{P}_{0} \Delta+\cdots$,

where $\Delta \ll R e^{-k}$ for any exponent $k>0$. There is a standard wall-layer region, spanned by $Y^{ \pm}=O(1)$, where $z= \pm 1 \mp Y^{ \pm} / R e^{1 / 2}$, in which the expansions are

$U=-1+\cdots+u_{0}^{ \pm}\left(Y^{ \pm}\right) \Delta+\cdots$,

$V=\mp 2 Y^{ \pm} R e^{-1 / 2}+\cdots+v_{0}^{ \pm}\left(Y^{ \pm}\right) \Delta R e^{-1 / 2}+\cdots$,

with $\mathrm{d} / \mathrm{d} z=\mp R e^{1 / 2} \mathrm{~d} / \mathrm{d} Y^{ \pm}$. The key difference here is that we are applying a straightforward $R e^{-1 / 2}$ wall-layer scaling, but it is to terms that are exponentially small.

The equations in this wall layer are

$u_{0}^{ \pm \prime \prime}=\bar{P}_{0}-2 u_{0}^{ \pm}+2 Y^{ \pm} u_{0}^{ \pm \prime}$,

$2 u_{0}^{ \pm} \pm v_{0}^{ \pm \prime}=0$,

to be solved subject to $u_{0}^{-}(0)=0, v_{0}^{-}(0)=0$ in the lower wall layer at $z=-1$ but $u_{0}^{+}(0)=1$ (corresponding to the imperfection in boundary conditions), $v_{0}^{+}(0)=0$ in the upper wall layer at $z=1$; here the prime notation denotes differentiation with respect to the appropriate argument $Y^{ \pm}$. The solution is found to be

$u_{0}^{ \pm}=\frac{1}{2}\left(\left(2 J^{ \pm}-\bar{P}_{0}\right)\left[\exp \left(Y^{ \pm 2}\right)-\sqrt{\pi} Y^{ \pm} \operatorname{erfi}\left(Y^{ \pm}\right)\right]+\bar{P}_{0}+4 C_{1} Y^{ \pm}\right)$

for some constant $C_{1}$ and $J^{+}=-1, J^{-}=0$, where $\operatorname{erfi}\left(Y^{ \pm}\right)=-i \operatorname{erf}\left(i Y^{ \pm}\right)$. Here $J^{ \pm}$plays the role of the imperfection, which is only applied at the upper wall. 
We note that as $\eta \rightarrow \pm \infty$ in the mid-plane shear layer,

$\tilde{U}_{s 1} \sim \frac{2 \eta}{c \mp 1}+\frac{1}{\sqrt{\pi}\left(c^{2}-1\right)} \frac{e^{-\eta^{2}}}{\eta^{2}}$,

while as $Y^{ \pm} \rightarrow \infty$ in the upper/lower wall layer, we find that

$u_{0}^{ \pm} \sim \frac{\bar{P}_{0}-2 J^{ \pm}}{4} \frac{e^{Y^{ \pm 2}}}{Y^{ \pm}}$.

This exponential growth on leaving the wall layers is inevitable and must be properly taken account of in the asymptotic solution.

The $\eta$ term in (17) matches to the $O(1)$ core-flow solution as $\eta \rightarrow \infty$; however, it seems that the only term that can (indirectly) match with the exponentially growing term in (18) is the corresponding decaying term in (17). This is consistent with our assumption that $\Delta \ll R e^{-k}$ for any exponent $k>0$ and suggests that we examine the intermediate $z=O(1)$ region in more detail.

\subsubsection{A WKB expansion in the interior regions}

We clearly cannot ignore the exponentially small terms and therefore, as discussed in the review article [10], the strategy is to isolate these terms so that they can be treated separately from the algebraic part of the asymptotic series. There are two interior regions, $c<z<1$ and $-1<z<c$, in which we denote quantities using the superscripts $(\cdot)^{+}$and $(\cdot)^{-}$, respectively.

In each interior layer, we introduce expansions of the form:

$U=U_{0}^{ \pm}(z)+U_{1}^{ \pm}(z) R e^{-1}+\cdots+U_{w}^{ \pm}(z) \Gamma^{ \pm}(R e, \Delta)+\cdots$,

with a similar expansion for $V$, where the gauge functions $\Gamma^{ \pm}(R e, \Delta) \ll 1$ are exponentially small, but their exact form is to be determined as part of the matching process.

The pressure perturbation $\Delta \bar{P}_{0}$ does not influence this region, and the governing equations for the unknowns $\left(U_{w}^{ \pm}, V_{w}^{ \pm}\right)$are

$$
\begin{aligned}
R e^{-1} U_{w}^{ \pm \prime \prime}= & 2\left[U_{0}^{ \pm}+R e^{-1} U_{1}^{ \pm}+\cdots\right] U_{w}^{ \pm} \\
& +\left[V_{0}^{ \pm}+R e^{-1} V_{1}^{ \pm}+\cdots\right] U_{w}^{ \pm \prime}+\left[U_{0}^{ \pm \prime}+R e^{-1} U_{1}^{ \pm \prime}+\cdots\right] V_{w}^{ \pm},
\end{aligned}
$$

$2 U_{w}+V_{w}^{\prime}=0$

where the bracketed terms are as provided by (6a)-(6b) and (11a)-(11b).

Solving via a WKB method is a somewhat lengthy but otherwise straightforward process, yielding the solutions:

$U_{w}^{+}(z)=\left\{\frac{R e}{(c-1)(c-z)^{2}(z-1)^{2}}+\cdots\right\} \exp \left(\operatorname{Re} F_{0}(z)+F_{1}(z)\right)$,

$U_{w}^{-}(z)=\left\{\frac{\operatorname{Re}}{(1+c)(c-z)^{2}(1+z)^{2}}+\cdots\right\} \exp \left(\operatorname{Re} G_{0}(z)+G_{1}(z)\right)$,

where $F_{0,1}$ and $G_{0,1}$ are not given explicitly here. However, to match the solutions together, we do require the limiting forms of these solutions as $z \rightarrow \pm 1$ and $z \rightarrow c^{ \pm}$.

As $z \rightarrow 1$ we find that

$F_{0}(z)=\frac{1-3 c}{3(c-1)}+(z-1)^{2}+O\left((z-1)^{3}\right)$,

$F_{1}(z)=-\frac{1}{3(c-1)^{3}(c+1)}+O\left((z-1)^{3}\right)$,

and as $z \rightarrow c^{+}$, 
$F_{0}(z)=\frac{c^{2}(c-3)}{3(c-1)}-(z-c)^{2}+O\left((z-c)^{3}\right)$,

$F_{1}(z)=-\frac{(c-1)^{3}+1}{3(c-1)^{3}(c+1)}+O(z-c)$.

As $z \rightarrow-1$ we find that

$G_{0}(z)=-\frac{3 c+1}{3(c+1)}+(z+1)^{2}+O\left((z+1)^{3}\right)$,

$G_{1}(z)=-\frac{1}{3(c+1)^{3}(c-1)}+O\left((z+1)^{3}\right)$,

and as $z \rightarrow c^{-}$,

$G_{0}(z)=\frac{c^{2}(c+3)}{3(c+1)}-(z-c)^{2}+O\left((z-c)^{3}\right)$,

$G_{1}(z)=\frac{(c+1)^{3}-1}{3(c+1)^{3}(c-1)}+O(z-c)$.

\subsubsection{Matching across the channel}

We now require that the series (19) in each core region matches to (14) on approaching the boundaries and (7) on approaching the shear layer. To achieve this, it only remains to match the exponentially small terms, ensuring that (21) matches to (18) as $z \rightarrow 1\left(Y^{+} \rightarrow \infty\right)$ and to (17) as $z \rightarrow c^{+}(\eta \rightarrow \infty)$, which leads to

$$
\frac{\Delta}{4 \operatorname{Re}}\left(\bar{P}_{0}+2\right)=\operatorname{Re} \Gamma^{+} \frac{1}{(c-1)^{3}} \exp \left\{-\frac{1-3 c}{3(1-c)} \operatorname{Re}-\frac{1}{3(c-1)^{3}(c+1)}\right\}
$$

(because $J^{+}=1$ ) and

$\frac{1}{R e^{3 / 2} \sqrt{\pi}} \frac{1}{c^{2}-1}=\operatorname{Re} \Gamma^{+} \frac{1}{(c-1)^{3}} \exp \left\{\frac{c^{2}(c-3)}{3(c-1)} R e-\frac{(c-1)^{3}+1}{3(c-1)^{3}(c+1)}\right\}$,

respectively. Similarly, in the lower region, we require that (21) matches to (18) as $z \rightarrow-1\left(Y^{-} \rightarrow \infty\right)$ and to (17) as $z \rightarrow c^{-}(\eta \rightarrow-\infty)$, which leads to

$$
\frac{\Delta \bar{P}_{0}}{4 \operatorname{Re}}=\operatorname{Re} \Gamma^{-} \frac{1}{(c+1)^{3}} \exp \left\{-\frac{3 c+1}{3(c+1)} \operatorname{Re}-\frac{1}{3(c+1)^{3}(c-1)}\right\},
$$

(because $J^{-}=0$ ) and

$\frac{1}{R e^{3 / 2} \sqrt{\pi}} \frac{1}{c^{2}-1}=\operatorname{Re} \Gamma^{-} \frac{1}{(c+1)^{3}} \exp \left\{\frac{c^{2}(c+3)}{3(c+1)} \operatorname{Re}+\frac{(c+1)^{3}-1}{3(c+1)^{3}(c-1)}\right\}$,

respectively.

The constraints (22b) and (23b) serve to define the functions $\Gamma^{ \pm}$in (19); they are both $O\left(R e^{-5 / 2} \exp \left(-\mu^{ \pm} R e\right)\right.$, where $\mu^{ \pm}$are positive constants, the values of which depend upon the location of the interior shear layer, $c$.

On eliminating in favour of $\Delta$ and $c$, under the assumption that $0<c<1$ corresponding to a shear layer that is displaced towards the 'upper' wall, we find that

$$
\Delta=\frac{2}{\left(c^{2}-1\right) \sqrt{\pi R e}} \exp \left\{-\frac{1}{3}\left((c-1)^{2} R e-\frac{1}{c+1}\right)\right\}
$$

a similar result obviously holds in the $-1<c<0$ case.

Figure 7 shows profiles of the radial component $U(z)$ for $R e=25,50,100$ and 200 as computed from the original governing equations. In these calculations the boundary conditions have a slight symmetry imperfection, 


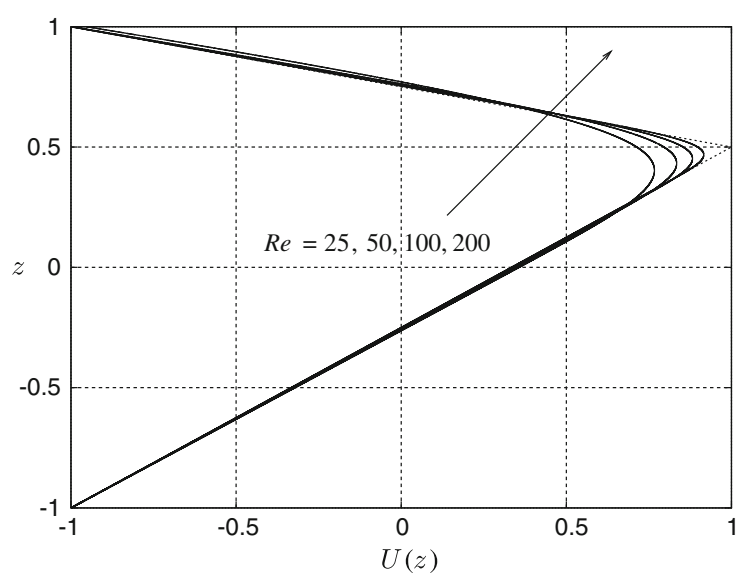

Fig. 7 A series of profiles for $U(z)$ (shown as solid lines) for $R e=25,50,100$, and 200. In this case, the upper boundary condition is $U(z=1)=-1-\Delta$, where $\Delta$ is chosen to induce an exponentially decreasing imperfection to the mid-plane symmetry by applying (24) with $c=0.5$. As can be seen, this exponentially decreasing imperfection leads to a persistent $O(1)$ effect on the radial velocity profile, it being skewed to peak at $z=c=0.5$ as $R e \rightarrow \infty$. The dashed lines show the leading-order inviscid solution confirming the matching process that determines the functional relation between $c$ and the imperfection $\Delta$. At the final value of $R e=200$, the symmetry imperfection in the boundary conditions is such that $|\Delta| \approx 7.7 \times 10^{-9}$

with $U(-1)=-1$ and $U(1)=-1-\Delta$. However, we have chosen $\Delta$ to satisfy (24) with $c$ specified to be $c=0.5$. As can be seen from the figure, the analysis above makes the correct predictions in that, for this functional form of $\Delta$, the numerical data approach the asymptotic description in a convincing manner. It is worth noting that at the final Reynolds number $(R e=200)$ shown, the imperfection in the symmetry of the boundary conditions is only slightly larger than the tolerance applied in the numerical scheme (in this case $|\Delta| \approx 7.7 \times 10^{-9}$ ). It is also worth emphasising yet again that the solution (when $\Delta=0$ ) is locally unique and symmetric at this Reynolds number (there is no bifurcation), and that the $O(1)$ asymmetry in the profile is purely a consequence of (indeed, forced by) this $10^{-9}$ imperfection. For a second-order finite difference scheme, accurate computation of the boundary value problem in this case, with $R e=200$, was performed with $8 \times 10^{4}$ nodal points in $z$.

\section{Linear perturbations to the mid-plane symmetric, self-similar, state}

In the high Reynolds number limit, to algebraic order in the asymptotic description, there is a continuum of states parameterised by $c \in[-1,1]$. It is only when terms that are exponentially small are included that the base flow profile, i.e. the value $c$, is determined. In the absence of any imperfection $(\Delta \equiv 0)$ we obtain the similarity solution that is symmetric about the channel midplane $(c=0)$. One might naturally wonder what the consequences of this 'near continuum' of states are in terms of the spatial and temporal stability of this symmetric self-similar state.

\subsection{Temporal modes}

For unsteady motion of this self-similar form, we simply augment the system (3) with the acceleration term $U_{t}$ in the radial momentum equation; it is well known that this stagnation form of solution is preserved for unsteady flows. We can therefore address the temporal stability of the perfect $(\Delta \equiv 0)$ base flow to linear perturbations of the same self-similar form by seeking a solution in the form

$$
\begin{aligned}
u(r, z, t) & =r\left(U(z)+\delta \tilde{u}(z) e^{s t}\right), \\
v(r, z, t) & =V(z)+\delta \tilde{v}(z) e^{s t}, \\
p(r, z, t) & =\frac{1}{2} r^{2} P+\frac{1}{A^{2}} Q(z)+\delta\left(\frac{1}{2} r^{2} \tilde{p}+\frac{1}{A^{2}} \tilde{q}(z)\right) e^{s t},
\end{aligned}
$$


where $\delta \ll 1$ and $\tilde{p}$ is a constant. The governing eigenvalue problem for the temporal growth rate $s$ is,

$$
\begin{aligned}
& s \tilde{u}+2 U \tilde{u}+V \tilde{u}^{\prime}+\tilde{v} U^{\prime}=-\tilde{p}+\frac{\tilde{u}^{\prime \prime}}{\operatorname{Re},} \\
& 2 \tilde{u}+\tilde{v}^{\prime}=0,
\end{aligned}
$$

where we have not given the decoupled equation for the pressure correction $\tilde{q}$ and the boundary conditions are $\tilde{u}=\tilde{v}=0$ on $z= \pm 1$.

Given the results of the previous section, we should expect at least one temporal (symmetry breaking) eigenmode to exist with $s$ exponentially small. Such modes must exist because one can construct a neutral solution to (26), accurate to all algebraic order, by considering the difference of two neighbouring states in the 'algebraic continuum' of base flows about $\Delta=0$. More formally, the matched asymptotic approach considered for the base flow can equally be applied to the perturbation equations (26), although we avoid most of the details here as they follow the method applied in the previous section. It is sufficient to note that the dominant eigenmode must have $\tilde{p} \equiv 0$ (for it to break the mid-plane symmetry of the base flow), and the core solution for $z \in[0,1]$ is

$\tilde{u}=1-z+O\left(R e^{-1}\right), \quad \tilde{v}=(z-1)^{2}+O\left(R e^{-1}\right)$,

with analogous expressions in the region $z \in[-1,0]$. This outer solution again (as for the base flow) satisfies the conditions at the wall, but a boundary layer region is still required for exponentially small terms.

We now consider the upper wall layer and, as before, introduce $z=1-Y^{+} R e^{-1 / 2}$ then the appropriate expansion is

$$
\begin{aligned}
u= & r\left([-1+\cdots]+\delta\left[Y^{+} R e^{-1 / 2}+\cdots+\gamma(R e) \tilde{u}_{0}^{+}\left(Y^{+}\right)+\cdots\right] e^{s t}\right), \\
v= & {\left[2 Y^{+} R e^{-1 / 2}+\cdots\right] } \\
& +\delta\left[Y^{+2} R e^{-1}+\cdots+\gamma(\operatorname{Re}) \operatorname{Re}^{-1 / 2} \tilde{v}_{0}^{+}\left(Y^{+}\right)+\cdots\right] e^{s t},
\end{aligned}
$$

where $\gamma(R e)$ is beyond all orders and to be determined by a matching process. The only significant question to address for this problem is: how does the exponentially small temporal growth rate appear in the eigenproblem? We can see from (28) that if

$s=s_{0} R e^{1 / 2} \gamma(R e)$,

then the governing system for the exponentially small components $\tilde{u}_{0}^{+}, \tilde{v}_{0}^{+}$is forced by an inhomogeneity, $s_{0} Y^{+}$, which then leads to exponential growth as $Y^{+} \rightarrow \infty$. This growth must be matched via a WKB solution in the core to the exponential terms in the shear layer around $z=0$ (as in the previous section). This results in a least damped temporal growth rate of

$s=O\left(\operatorname{Re} \exp \left(-\frac{R e}{3}\right)\right)$.

As $s$ remains real, we may view this as the base flow being exponentially close to possessing a symmetry-breaking pitchfork bifurcation; however, no zero of $s$ is ever achieved. Indeed, there is no evidence of any other eigenmode becoming unstable, therefore this 'exponentially close to neutral' mode is the most relevant and will dominate the unsteady dynamics of this flow for increasing values of $R e$.

In Fig. 8, we present a numerical computation of the temporal growth rate $s$, scaled by the large Reynolds number behaviour (30). To determine $s$ numerically, we first discretise (26) to obtain the full discrete spectrum by solving with a QZ algorithm, before subsequently refining the solution with a much greater numerical resolution for the 'least damped' mode by a local refinement procedure (effectively solving (26) as a nonlinear problem with $s$ as an unknown).

Any transient (mid-plane) symmetry-breaking perturbation of the self-similar system will therefore require a time scale of $O\left(R e^{-1} \exp (R e / 3)\right)$ to decay. Nonlinear computations of the unsteady form of the self-similar equations readily show this exponentially long persistence of asymmetry, which is entirely in line with this eigenvalue analysis. We do not present such results here, and prefer instead to concentrate on the spatial analogue of this analysis. 
Fig. 8 Eigenvalues for the spatial ( $\lambda$, upper line $)$ and temporal $(s$, lower line $)$ growth rates, scaled by the large Reynolds number behaviour

$(s, \lambda \sim R e \exp (-\operatorname{Re} / 3))$.

These are spatial/temporal eigenmodes that are exponentially close to neutral and are both real valued

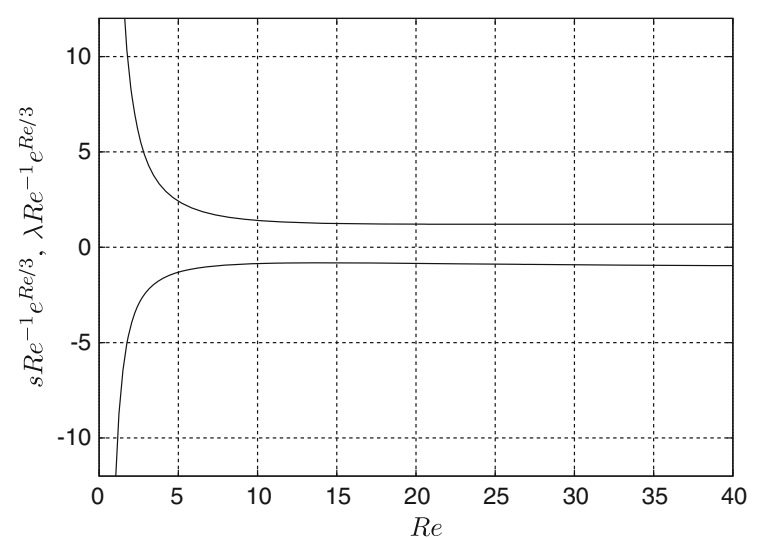

\subsection{Spatial modes}

For spatial eigenmodes of the symmetric $(\Delta=0)$ base flow, we seek an expansion of the form:

$$
\begin{aligned}
& u(r, z, t)=r U(z)+\delta r^{1+\lambda} \tilde{u}(z), \\
& v(r, z, t)=V(z)+\delta r^{\lambda} \tilde{v}(z), \\
& p(r, z, t)=\frac{1}{2} r^{2} P+\frac{1}{A^{2}} Q(z)+\delta\left(\frac{1}{2} r^{2+\lambda} \tilde{p}+\frac{r^{\lambda}}{A^{2}} \tilde{q}(z)\right),
\end{aligned}
$$

where $\delta \ll 1$, and $\tilde{p}$ is a constant. The governing eigenvalue problem for the spatial growth 'rate' $\lambda$, under appropriate assumptions, is

$$
\begin{aligned}
& (2+\lambda) U \tilde{u}+V \tilde{u}^{\prime}+\tilde{v} U^{\prime}=-\frac{1}{2}(2+\lambda) \tilde{p}+\frac{\tilde{u}^{\prime \prime}}{R e}, \\
& (2+\lambda) \tilde{u}+\tilde{v}^{\prime}=0,
\end{aligned}
$$

where we have not given the decoupled equation for the pressure $\tilde{q}$ and the boundary conditions are $\tilde{u}=\tilde{v}=0$ on $z= \pm 1$. We say 'under appropriate assumptions' here because, although the base flow $(U, V)$ is an exact reduction of the Navier-Stokes equations, the equations for a spatial perturbation are obtained in the large aspect ratio limit. In the derivation of (32), we have neglected terms of the form $A^{-2} r^{-2}$ (for example), where $A=R / L$ is the aspect ratio. The large-aspect ratio limit parabolises the perturbation equations, leading to a linear-eigenvalue problem, which then has eigenmodes of the form: (31).

Precisely as discussed for the temporal modes, we find that in the large Reynolds number limit, the least damped spatial growth rate is exponentially small, such that

$\lambda=O\left(R e \exp \left(-\frac{R e}{3}\right)\right)$.

In Fig. 8, we also present results from a numerical computation of the 'least damped' spatial eigenvalue $\lambda$, scaled by the large Reynolds number behaviour (33).

\section{Nonlinear, steady, finite-domain solutions}

When the flow domain is finite in its radial extent, we must solve the full axisymmetric Navier-Stokes equations (1) with an additional condition at the edge of the boundary $(r=1)$. To address the relevance of the previous asymptotic discussion to 'real' flows that are necessarily finite in radial extent, we shall find steady solutions to (1) numerically, subject to the boundary conditions: 


$$
\begin{aligned}
& v(r, z=-1)=0, \quad v(r, z=1)=0, \\
& u(r, z=-1)=-r, \quad u(r, z=1)=-r\left(1+\Delta\left(1-e^{A(r-1)}\right)\right), \\
& u(r=0, z)=0, \quad v_{r}(r=0, z)=0, \\
& u(r=1, z)=U_{c=0}(z ; \operatorname{Re})+\epsilon \sin (\pi z), \quad v_{r}(r=1, z)=0 .
\end{aligned}
$$

Here $\Delta$ is a measure of asymmetry in the lateral wall boundary conditions, $\epsilon$ is a measure of asymmetry at the domain's edge, and $U_{c=0}$ is the mid-plane symmetric similarity solution. Given that $U_{c=0}(z=1)=-1$ the boundary conditions at the corner position $r=1, z=1$ are self-consistent for any $O(1)$ value of the perturbation amplitudes $\epsilon$ and $\Delta$.

We tackle the problem using a Galerkin finite-element method for a given Reynolds number $R e$ and aspect ratio $A=R / L$. The cylindrical domain is decomposed into isoparametric, axisymmetric $Q_{2} P_{1}$ finite elements; see [11]. The pressure is discontinuous across element boundaries and is approximated by piecewise bi-linear polynomials. The two global coordinates $(r, z)$ and the two velocity components $(u, v)$ (in the meridional plane) are continuous across element boundaries and are approximated by piecewise bi-quadratic polynomials. The finite-element method is used to calculate approximate solutions to the weak form of the Navier-Stokes equations, as obtained by weighting the equations (1) by suitable test functions and integrating over the fluid domain. A consequence of the assumption of axisymmetric flow is that we can integrate over the azimuthal coordinate analytically. The resulting numerical problem is formulated and solved using the oomph-lib library [12].

If $\epsilon=\Delta=0$, then the symmetric similarity solution remains a solution of the finite-domain problem for all radii and we use this to validate the Navier-Stokes solver over a range of $R e$ and spatial resolutions. If $\Delta \neq 0$, then we are imposing an asymmetry in the lateral boundary conditions, in a manner analogous to that considered for the self-similar flow in Sects. 2 and 3. When $\epsilon \neq 0$, we are imposing a perturbation at the edge of the domain that breaks the (mid-plane) symmetry of the base flow. The choice of $\sin (\pi z)$ as a perturbation ensures that the mass flux into the domain remains zero as clearly must be the case; other symmetry-breaking mass-flux-conserving perturbations were investigated and they did not alter the qualitative features of the results that we present herein. Some care is required to ensure that the net mass flux of the similarity solution $U_{c=0}(z)$ is less than the global tolerance for the scheme when interpolated over the chosen spatial discretisation; this is most simply achieved by solving the boundary-value problem for $U_{c=0}(z)$ on precisely the same $z$-distribution of nodal points as will be applied to the full two-dimensional (2D)computation.

\subsection{Asymmetry in the stretching rates: $\epsilon=0, \Delta=0.01,0.05$}

We begin by computing solutions for which there is a peak 1 or $5 \%$ asymmetry in the lateral wall boundary conditions (i.e. $\Delta=0.01,0.05$ ), but for which symmetry is still imposed at the edge of the domain (i.e. $\epsilon=0$ ). The results of Sect. 2 demonstrate that the similarity solution becomes increasingly sensitive to such asymmetry as $R e$ is increased, and the description of Sect. 3 showed that the origin of this sensitivity is the exponential terms in the asymptotic description.

To assess the relevance of the self-similar results to the finite domain, we compute 2D solutions over a range of parameters $R e \in[0,20], A \in[1,120]$ and compare the resulting dimensionless transverse flow velocity at the mid-plane, on the axis of rotational symmetry: $v(r=0, z=0)$. For a mid-plane symmetric solution, we must obviously have $v(r=0, z=0)=0$, but we find that the asymmetry in the stretching rate of the plates does lead to an increasingly asymmetric flow as $R e$ is increased. In Fig. 9, we show the predicted self-similar behaviour and the results from the 2D solution as the aspect ratio of the domain is increased to be $A=30,60,120$.

As the length of the domain is increased, for sufficiently large symmetry perturbations the predicted self-similar behaviour is recovered near the axis of rotational symmetry (see the $\Delta=0.05$ data set). However, for smaller values of $\Delta$, the approach to the self-similar predictions is extremely slow for increasing aspect ratios $A$. Given the results of Sect. 4, we should perhaps not be surprised that, as the Reynolds number becomes large enough for the exponential sensitivity in lateral boundary conditions to be felt, the exponentially small spatial eigenvalue leads to 


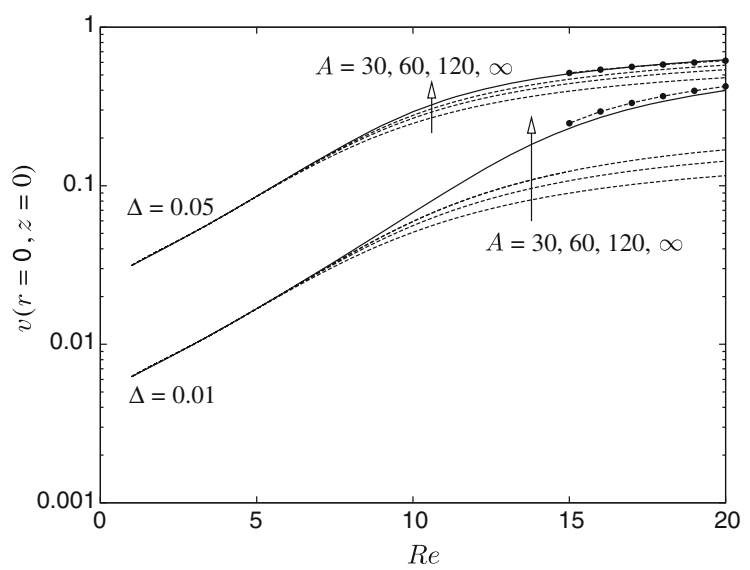

Fig. 9 The dependence of the transverse flow for a finite-domain solution, as measured at the mid-plane of the channel $(z=0)$, on the axis of rotational symmetry $(r=0)$. We show the data for both a 1 and a $5 \%$ asymmetry in stretching rates $(\Delta=0.01$ and 0.05 , with $\epsilon=0)$ and aspect ratios of $A=30,60$ and 120 as dashed lines. The data set for a radially unbounded case $(A=\infty)$ is the similarity solution, shown as solid lines. The asymptotic predictions of Sect. 3 are shown for $R e \geq 15$ by the data points

the flow near the origin being more strongly influenced by the boundary condition at the edge of the domain unless the aspect ratio is very large. This motivates us to consider the case of an asymmetry induced by the 'far field' $\left(r^{*}=R\right.$ ) boundary condition (with $\Delta=0, \epsilon \neq 0$ ).

5.2 Asymmetry at the domain's edge: $\epsilon=0.01,0.05,0.25, \Delta=0$

We now impose an asymmetry at the edge of the domain by varying $\epsilon$ with $\Delta=0$ in the conditions (34). As a metric of the response of the finite-domain solution, we examine the difference between the symmetric similarity solution and the finite-domain numerical solution:

$\phi(r)=\left(u(r, z=1 / 2)-r U_{c=0}(z=1 / 2)\right)(\epsilon r)^{-1}$;

where $z=1 / 2$ is an arbitrary choice of position for the measure. We have normalised this measure such that $\phi(r=1)=1$ and show the radial development of this difference for $\epsilon=0.01 \mathrm{in} \mathrm{Fig.} \mathrm{10.} \mathrm{The} \mathrm{figure} \mathrm{shows} \mathrm{the} \mathrm{radial}$ decay of the asymmetry imposed at the domain's edge for $R e=2.5,5,10,20$ and aspect ratios $A=15,30,60$. As we have already discussed in Sect. 4, a linearised mid-plane symmetry breaking perturbation in the limit of large aspect ratio $A \gg 1$ has a radial dependence of $r^{1+\lambda}$ (for the radial velocity perturbation) where $\lambda \sim \operatorname{Re} \exp (-\operatorname{Re} / 3$ ). Figure 10 demonstrates that, even at an aspect ratio of $A=60$, a Reynolds number of $R e=20$ is sufficient for a perturbation at the edge of the channel to be maintained across the entire length of the domain all the way to $r=0$.

In Fig. 11, we repeat the metric of Fig. 10 but increase the amplitude of the symmetry perturbation by increasing $\epsilon$ to be $0.01,0.05$, and 0.25 with $A=60$. We observe that there is little qualitative difference and the dominance of the edge condition along the entire length of the channel is maintained even for strongly nonlinear perturbations.

Finally, in Fig. 12, we compare the predictions for the spatial decay rate of perturbations to the symmetric selfsimilar state as $A \rightarrow \infty$ with the results of a finite-domain computation at $A=60, \operatorname{Re}=10,15,20$ and $\epsilon=0.01$. The spatial analysis of Sect. 4 indicates that, for $\epsilon \ll 1, A \gg 1$, perturbations to the self-similar base state are such that the difference measure (35) has a predicted radial dependence of

$\phi(r) \sim r^{\lambda}$,

when dominated by a single spatial eigenmode. A local spatial decay coefficient for $2 \mathrm{D}$ states may therefore be defined by

$\hat{\lambda}(r)=\frac{r \phi^{\prime}(r)}{\phi(r)}$. 


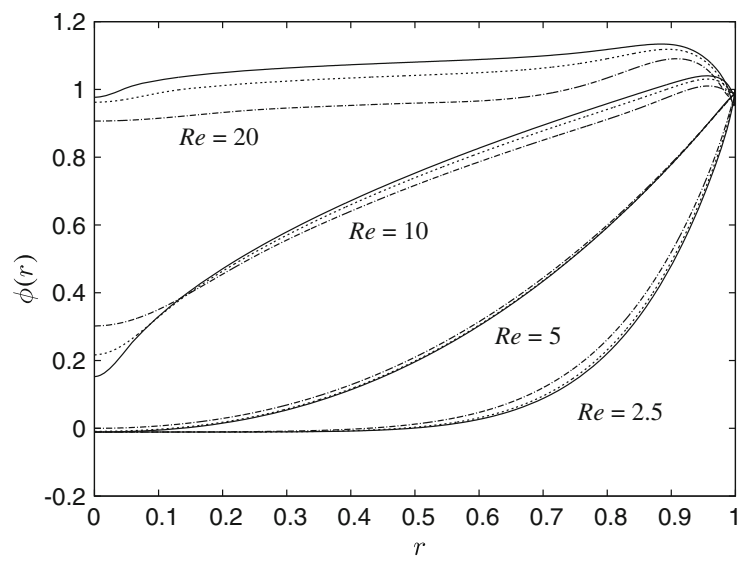

Fig. 10 The distribution of $\phi(r)$, which measures the difference between a finite domain non-self-similar solution and the self-similar solution, shown for increasing values of $R e$ and $A$. The finite domain states have an asymmetry imposed at the domain's edge with $\epsilon=0.01, \Delta=0$ in the boundary conditions (34). The cross sections are presented at $R e=2.5,5,10$ and 20 and at aspect ratios of $A=15$ (dash-dot) $A=30$ (dashed) and $A=60$ (solid)

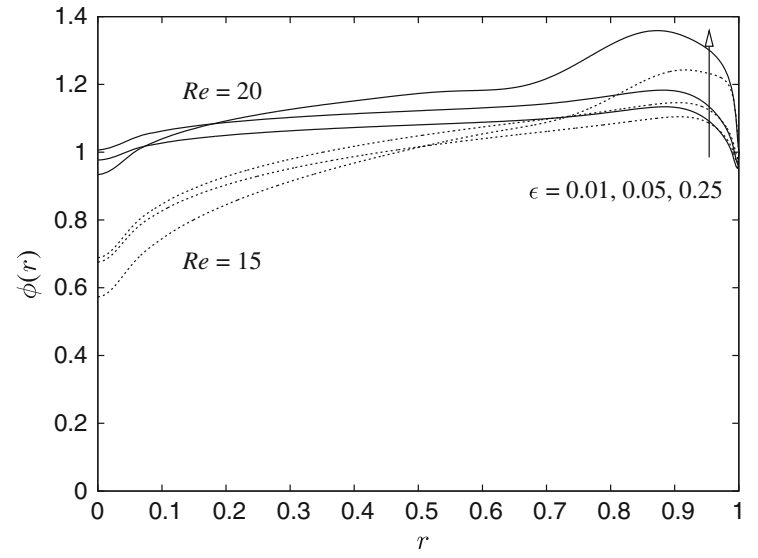

Fig. 11 The distribution of $\phi(r)$, which is a measure of the difference between a finite domain non-self-similar solution and the self-similar solution, shown for increasing values of $\epsilon$ with $R e=15,20, A=60$ and $\Delta=0$. Even for strongly nonlinear asymmetry perturbations at the domain's edge, the flow response within the channel is qualitatively similar

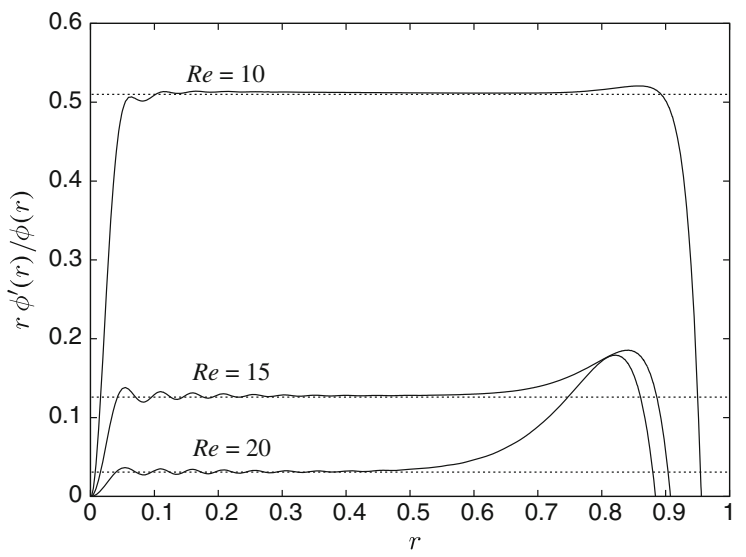

Fig. 12 A comparison of the spatial decay rate as predicted in Sect. 4 and the local decay rate of a finite-domain non-self-similar state for an imposed asymmetry at the domain's edge with $\epsilon=0.01, \Delta=0$ and $A=60$. For linearised spatial perturbations, we found that $\phi \sim r^{\lambda}$, and can therefore compare our predicted decay rate $\lambda$ for $A \gg 1$ with the local value $r \phi^{\prime} / \phi$ along the length of the channel. The corresponding values of $\lambda$ predicted in Sect. 4 are shown as the broken horizontal lines

Figure 12 compares the local decay coefficient (37) with the linearised, infinite domain, eigenvalue $\lambda$ (see Fig. 8) showing excellent agreement, with the modes rapidly (exponentially) becoming spatially neutral as the Reynolds number is increased.

\section{Conclusions}

In this study, we have considered a well-known exact solution of the Navier-Stokes equations in a radially unbounded flow and shown that, despite being an innocuous boundary-value problem, there is some subtlety in the solution structure for large values of the Reynolds number. An accurate description of the $R e \gg 1$ limit is only possible if one includes terms in the asymptotic series that are exponentially small in $R e$. 
That the terms beyond all orders in the $R e \gg 1$ asymptotic series are important has two consequences: (i) in the context of the boundary-value problem for the exact solution, an exponentially small asymmetry in the boundary conditions leads to $O(1)$ effects, and (ii) there are linear spatial and temporal eigenmodes that are exponentially close to being neutral. For the exponential sensitivity in the boundary-value problem, we have demonstrated that when $R e \gg 1$ a symmetry imperfection of $O\left(R e^{-\frac{1}{2}} \exp (-\mu R e)\right)$, where $0<\mu<\frac{1}{3}$, will still have an $O(1)$ influence. With regards to linearised perturbations, we have shown that (mid-plane) symmetry-breaking unsteady disturbances to the self-similar flow will decay like $\exp (s t)$, where $s<0$ and $s=O(\operatorname{Re} \exp (-\operatorname{Re} / 3))$. Similarly, spatial disturbances behave like $r^{\lambda}$, relative to the base flow, where $\lambda>0$ and $\lambda=O(\operatorname{Re} \exp (-\operatorname{Re} / 3))$. In both cases, the near neutral modes are the ones that dominate the spatio-temporal behaviour.

As the base flow described in this article is an 'exact solution', all of the features discussed in the context of the boundary-value problem are necessarily also present in the Navier-Stokes equations, provided that the 'far field' (i.e. the solution for large-radius) in any problem is consistent with the similarity solution. However, in practice, one has little control over such far-field conditions, and so we have also cast the results for these self-similar flows into a more practical context by performing Navier-Stokes computations over a finite domain (with the radius of the domain being up to 120 times longer than the channel half-width). Although the self-similar solutions can describe the near-axis $r \ll 1$ finite domain solution in some cases (see Fig. 9 with $\Delta=0.05$ for example) it appears that the spatial stability results are of most practical significance. As the Reynolds number is increased, spatial growth rates become exponentially close to neutral which (in practice) leads to any asymmetry in the 'far field' affecting the solution across the entire radial extent of the channel, rather than being localised near the edge of the domain. Of course one can always choose a domain that is sufficiently long for the perturbation to decay, but owing to the exponentially decreasing nature of the eigenvalue $\lambda$, the (exponentially long) lengths of channel required rapidly make little practical sense.

Given the intricate nature of this analysis, and that the linear stability results show eigenvalues that are exponentially close to being neutral, one may suspect that the problem would not have to be altered much to give rise to a bifurcation. One could, for example (as suggested by a referee of this study) seek modes that are 3D and/or time-periodic; however, we suggest an alternative scenario that may give rise to a robust structure even within the confines of the steady axisymmetric formulation. Even for moderate Reynolds numbers (based on the channel half-height), as the aspect ratio is increased, the local flow speed will also increase linearly with radius. It seems highly likely therefore that the solution space must eventually become more complex and potentially nonunique as a local Reynolds number based on radius (rather than channel half-height) increases. A likely scenario is therefore that in practice, for a moderate $R e$ (as defined herein), there will be an aspect ratio for which instability develops beyond a critical radius in the channel. It is this large-radius instability that will then provide a (mid-plane) symmetry-breaking perturbation, which will then inevitably affect the entire channel length through the existence of near-neutral spatial modes. Obviously, such modes could only be captured by a detailed computational examination of the $2 \mathrm{D}$ eigenvalue problem over the parameter spaced spanned by the Reynolds number and aspect ratio.

\section{References}

1. Berman AS (1953) Laminar flow in channels with porous walls. J Appl Phys 24:1232-1235

2. Terrill RM (1966) Flow through a porous annulus. Appl Sci Res 17:204-222

3. von Kármán T (1921) Über laminare und turbulente Reibung. Z Angew Math 1:233-252

4. Brady JF, Acrivos A (1981) Steady flow in a channel or tube with an accelerating surface velocity. An exact solution to the Navier-Stokes equations with reverse flow. J Fluid Mech 112:127-150

5. Riley N, Drazin PG (2006) The Navier-Stokes equations: a classification of flows and exact solutions. London Mathematical Society lecture note series 334. Cambridge University Press, Cambridge

6. Hall P, Papageorgiou DT (1999) The onset of chaos in a class of exact Navier-Stokes solutions. J Fluid Mech 393:59-87

7. Robinson WA (1976) The existence of multiple solutions for the laminar flow in a uniformly porous channel with suction at both walls. J Eng Math 10:23-40

8. Cox SM, King AC (1997) On the asymptotic solution of a high-order nonlinear ordinary differential equation. Proc Roy Soc Lond A $453: 711-728$ 
9. Terrill RM (1973) On some exponentially small terms arising in flow through a porous pipe. QJMAM 26:347-354

10. Boyd JP (1999) The devil's invention: asymptotic, superasymptotic and hyperasymptotic series. Acta Appl Math 56:1-98

11. Gresho PM, Sani RL (1998) Incompressible flow and the finite element method. Wiley, Chichester

12. Heil M, Hazel AL (2006) oomph-lib: An object-oriented multi-physics finite-element library. In: Schafer M, Bungartz H-J (eds.) Fluid-structure interaction. Springer, New York 